\title{
Differentiation of functional gastrointestinal disorders from healthy volunteers by lactulose hydrogen breath test and test meal
}

\author{
Schindler, Valeria ; Giezendanner, Stéphanie ; Bütikofer, Simon ; Murray, Fritz ; Runggaldier, Daniel ; \\ Schnurre, Larissa ; Zweig, Annina ; Fried, Michael ; Pohl, Daniel
}

\begin{abstract}
BACKGROUND AND AIM Functional dyspepsia (FD) is a common disorder of gut-brain interaction with incompletely understood pathophysiology. Consequently, heterogeneous expert opinions on diagnostic tests and assessment of treatment efficacies exist. So far, no consensus about the most relevant diagnostic and outcome tool has been reached. In this study, we aimed to analyze the significance of a combined lactulose hydrogen breath test (LHBT) and liquid meal, yet representing a standardized test in irritable bowel syndrome (IBS), in FD. METHODS We analyzed data of 146 FD, 204 IBS patients, and 50 healthy volunteers (HV). All patients underwent LHBT with a meal-drink consisting of 30-g Lactulose and 400-mL Ensure®. Effect of abdominal symptom generation in FD/IBS compared with HV was assessed on a patient-reported Likert-scale. RESULTS There was a significant difference between FD/IBS patients and HV in LHBT-induced abdominal pain (odds ratio [OR] 246.9, 95\% confidence interval [CI] 26.6-2290.7; OR 161.2, 95\% CI 16.9-1534.8), abdominal bloating (OR 384.8, 95\% CI 92.92135.4; OR 524.1, 95\% CI 114.7-3432.3), borborygmi (OR 9.9, 95\% CI 2.2-46.9; OR 17.7, 95\% CI 4.7-67.4), nausea only in FD (OR 174.4, 95\% CI 15.5-5375.5), and diarrhea in IBS only (OR 25.8, 95\% CI 2.0-7012.6). Hydrogen production was not significantly different in FD/IBS and HV. CONCLUSIONS In this study, we demonstrated significant differences in postprandial symptom generation in FD and IBS compared with HV after LHBT. This does not only allow us to discriminate FD/IBS from HV but may also represent a diagnostic and monitoring tool for FD/IBS in the future, including monitoring of treatment effects.
\end{abstract}

DOI: https://doi.org/10.1111/jgh.14551

Posted at the Zurich Open Repository and Archive, University of Zurich

ZORA URL: https://doi.org/10.5167/uzh-161660

Journal Article

Accepted Version

Originally published at:

Schindler, Valeria; Giezendanner, Stéphanie; Bütikofer, Simon; Murray, Fritz; Runggaldier, Daniel; Schnurre, Larissa; Zweig, Annina; Fried, Michael; Pohl, Daniel (2019). Differentiation of functional gastrointestinal disorders from healthy volunteers by lactulose hydrogen breath test and test meal. Journal of Gastroenterology and Hepatology, 34(5):843-851.

DOI: https://doi.org/10.1111/jgh.14551 


\title{
Differentiation of functional gastrointestinal disorders from healthy volunteers by lactulose hydrogen breath test and test meal
}

\section{Short title:}

\section{LHBT allows discrimination between FGID and HV}

\begin{abstract}
Valeria Schindler ${ }^{1}$, Stéphanie Giezendanner ${ }^{2}$, Simon Bütikofer ${ }^{1}$, Fritz Murray ${ }^{1}$, Daniel Runggaldier ${ }^{3}$, Larissa Schnurre ${ }^{1}$, Annina Zweig ${ }^{1}$, Michael Fried ${ }^{1}$, Daniel Pohl ${ }^{1}$

${ }^{1}$ Division of Gastroenterology and Hepatology, University Hospital Zurich, Zurich, Switzerland; ${ }^{2}$ Centre for Primary Health Care, University of Basel, Basel, Switzerland; ${ }^{3}$ Department of Otorhinolaryngology, University Hospital Zurich, Zurich,
\end{abstract}

Switzerland

Corresponding author:

Dr. med. Valeria Schindler

Division of Gastroenterology and Hepatology

University Hospital Zurich

Raemistrasse 100

8091 Zurich

Switzerland

E-Mail: valeria.schindler@usz.ch

Tel: +412551658 or +412551111

Author contributions:

Valeria Schindler: performed the research, collected and analysed data, and wrote the paper. Stéphanie Giezendanner: designed the statistics, analysed data, approved manuscript. Simon Bütikofer: performed research, collected data, approved manuscript. Fritz Murray: performed research, collected data, approved manuscript. Daniel Runggaldier: collected and analysed data, approved manuscript. Larissa Schnurre: performed research, collected data, approved manuscript. Annina Zweig: collected data, approved manuscript. Michael Fried: contributed to study design, approved manuscript. Daniel Pohl: designed the study, performed the research, revised and approved manuscript

\section{Funding/Acknowledgement:}

This study was supported by Swiss National Foundation (SNF) grant 320030_166670

Conflicts of interest:

Valeria Schindler: support from Swiss National Foundation (SNF, grant funding) for research but no financial relationships or activities with any organizations that might have an interest in the submitted work; Stéphanie Giezendanner: none; Simon Bütikofer: none; Fritz Murray: none; Larissa Schnurre: none; Daniel Runggaldier: none; Annina Zweig: none; Michael Fried: none; Daniel Pohl: none 


\section{Background and Aim}

Functional dyspepsia (FD) is a common disorder of gut-brain interaction with incompletely understood pathophysiology. Consequently, heterogenous expert opinions on diagnostic tests and assessment of treatment efficacies exist. So far, no consensus about the most relevant diagnostic and outcome tool has been reached. In this study we aimed to analyze the significance of a combined lactulose hydrogen breath test and liquid meal (LHBT), yet representing a standardized test in IBS, in FD.

\section{Methods}

We analyzed data of 146 FD, 204 IBS patients and 50 HV. All patients underwent LHBT with a meal-drink consisting of $30 \mathrm{~g}$ Lactulose and $400 \mathrm{ml}$ Ensure ${ }^{\circledR}$. Effect of abdominal symptom generation in FD/IBS compared to HV was assessed on a patient-reported Likert-scale.

\section{Results}

There was a significant difference between FD/IBS-patients and HV in LHBT induced abdominal pain (OR 246.9, 95\% Cl 26.6-2290.7 / OR 161.2, 95\% Cl 16.9-1534.8), abdominal bloating (OR 384.8, 95\% Cl 92.9-2135.4 / OR 524.1, 95\% Cl 114.7-3432.3), borborygmi (OR 9.9, 95\% Cl 2.2-46.9 / OR 17.7, 95\% Cl 4.7-67.4), nausea only in FD (OR 174.4, 95\% Cl 15.5-5375.5) and diarrhea in IBS-only (OR 25.8, 95\% Cl 2.0-7012.6). Hydrogen production was not significantly different in FD/IBS and HV.

\section{Conclusions}

In this study we demonstrated significant differences in postprandial symptom generation in FD and IBS compared to HV after LHBT. This does not only allow us to discriminate FD/IBS from HV but may also represent a diagnostic and monitoring tool for FD/IBS in the future, including monitoring of treatment effects. 


\section{Introduction:}

Functional dyspepsia (FD) is a highly prevalent disorder of gut-brain interaction (FGID) with incompletely understood pathophysiology. As a consequence, heterogenous expert opinions on diagnostic tests and assessment of treatment efficacies exist. So far, no general consensus about the most relevant diagnostic and outcome tools has been reached.

FD is defined according to Rome IV criteria (1) as bothersome postprandial fullness and/or bothersome early satiation and/or bothersome epigastric pain and/or bothersome epigastric burning in combination with no evidence of structural disease that is likely to explain the symptoms. Additionally, FD patients often report symptoms like nausea and upper abdominal bloating after food intake $(2,3)$. Clinical and preclinical tests in FD patients range from gastric emptying/accommodation studies to Helicobacter pylori, duodenal biopsies, environmental/nutritional analyses, psychological questionnaires as well as hydrogen tests. Even though hydrogen breath tests in patients with FGIDs have been discussed controversially in the past years, they are stated to be useful in the diagnosis of carbohydrate maldigestion syndromes and the assessment of conditions associated with bloating as stated in the recently released North American Consensus on Breath Testing in gastrointestinal (GI) disorders (4). Unfortunately, wide heterogeneity in test execution and analysis has hindered comparisons and acceptance of results. The clinical significance of test results and therapeutic consequences often remain ambiguous. Also, frequency of breath sampling, test duration, dose and choice of test substance vary between institutions.

Among multiple studies analyzing lactulose hydrogen breath tests, Le Neve et al. (5) demonstrated differences in symptom response following a combined lactulose nutrient breath test (LHBT) between patients with irritable bowel syndrome (IBS) and healthy controls. Using this test made up of a standardized liquid meal in combination with fermentable carbohydrate lactulose, Pohl et al. recently showed differences in postprandial symptom generation in patients with IBS versus patients with combined IBS-FD and healthy controls (6). As a consequence of above mentioned findings, we hypothesized that not only IBS or IBS-FD but also FD-only patients may be discriminated from healthy volunteers (HV) based on abdominal symptoms and hydrogen values. The aim of this study was therefore to discriminate FD patients from HV based on the LHBT originally developed for IBS patients. 


\section{Methods}

\section{Design and study population}

We prospectively collected data of $50 \mathrm{HV}$ with signed informed consent, which responded to an advertisement. Inclusion criteria were age between 18-60 years, no antibiotic treatment 4 weeks prior to LHBT and no known gastrointestinal disorder (including FD and IBS) as well as lack of prior abdominal surgery. Data of HV were collected by physicians interviewing HV.

Between January 2015 and November 2017 data of 146 consecutive patients with FD (FD-only and FD-IBS mixed type) and 204 patients with IBS (IBS-only) who underwent LHBT as part of their routine work-up in our laboratory were analyzed. Most of the patients were part of our specialized functional gastrointestinal ambulatory clinic and only a few patients were referred from outside. All patients had prior endoscopy excluding structural disorders as well as $\mathrm{H}$. pylori infection or GI ulcera. Inclusion criteria were general informed consent, age older than 18 and FD and/or IBS diagnosed according to Rome IV, respectively Rome III criteria $(1,7)$.

\section{Lactulose hydrogen breath testing}

HV, FD and IBS patients were advised to a fasting period of at least 6-8 hours (incl. smoking and chewing gum) before LHBT in line with recommendations of the North American Breath Testing Consensus (4). LHBT was performed with Breathalyzer-HydroCheck (NEOMED Medizintechnik $\mathrm{GmbH}$ ) after mouth rinse with $15-20 \mathrm{ml}$ Drossadin Solution $0.1 \%$ for 30 seconds. Two baseline hydrogen measurements were performed and expressed in parts per million (ppm) and five baseline abdominal symptoms (nausea, abdominal pain, borborygmi, abdominal bloating and diarrhea) were recorded before ingestion of $400 \mathrm{ml}$ Ensure ${ }^{\circledR}$ (600 kcal, $20 \mathrm{~g}$ fat, $81 \mathrm{~g}$ carbohydrate, $25 \mathrm{~g}$ protein) and $30 \mathrm{~g}$ Lactulose. Hydrogen values and abdominal symptoms were collected in 10 min intervals for a total of 180 minutes. Abdominal symptoms were assessed on a 5-point Likert scale from 0 to 4 with 0 corresponding to no symptom and 4 resembling very strong symptoms. 


\section{Statistical analysis}

Data of continuous variables with Gaussian distribution are presented as means \pm standard deviation (SD) and as median and range in case of other than normal distribution. KruskalWallis-, t-test, ANOVA, Chi-squared and fisher exact test were used to compare demographics between healthy volunteers and FD patients. Mixed-effects models were used to assess group effects using the function "Ime" from the R package "nlme" for hydrogen and "glmer" from the R package "Ime4" for abdominal symptoms. Further, a nonparametric analysis of the longitudinal data was performed testing the relative effect of factor group using the function "f1.Id.f1" and the ANOVA-type statistic of the R package "nparLD". Age and sex were entered into multivariable models as covariates of no interest. A p-value $<0.05$ was considered statistically significant.

For the ROC, an equally sized test and training data set were created, drawing a random sample from the HV and the respective patient group (all patients, FD, FD-only, FD-IBS, IBSonly). After fitting a model with the training data set, the model parameters were used to predict the status (HV or patient) with the test data set. Subsequently, a ROC-GLM curve and a confusion matrix were produced.

Statistical analyses were performed with $R$ Version 3.2.0 ( $R$ Foundation for Statistical Computing, Vienna, Austria). Zurich Ethical Committee (BASEC-Nr. 2016-01887) approved the study.

\section{Results}

\section{Patient characteristics}

Of 350 patients 34 had FD-only, 112 had overlap FD-IBS and 204 patients suffered from IBSonly. The majority of patients were female ( $71.9 \%$ vs. $28.1 \%)$, which was also true in both subgroups (FD-only 24 females [70.6\%], FD-IBS subgroup 81 females [72.3\%], IBS-only 141 females [69.1\%]). In HV the female to male ratio was 33 to 17 (66.0\% vs. $34.0 \%$ ) in accordance with the general gender distribution of this entity. Median age in FD was 34 years (ys) (range 18-75ys), in FD-only 37 (range 21-71ys), in FD-IBS 34 (range 18-75ys), in IBS-only 36 (range 1877ys) and in HV 25 (range 18-58ys) ( $p<0.001)$. 


\section{Abdominal Symptoms}

\section{Group effect}

There was a significant difference between patients with FD and HV in LHBT induced nausea (OR 174.4, 95\% Cl 15.5-5375.5), abdominal pain (OR 246.9, 95\% Cl 26.6-2290.7), abdominal bloating (OR 384.8, 95\% Cl 92.9-2135.4) and borborygmi (OR 9.9, 95\% Cl 2.2-46.9). Effects remained significant after adjustment for age and gender. Only the occurrence of diarrhea did not significantly differ between the two groups (OR 12.3, 95\% $\mathrm{Cl} 0.2-970.9$ ) figure 1-5

In the subgroup of FD-only compared to HV, there was a significant difference in LHBT induced nausea (OR 171.5, 95\% Cl 28.1-2203.5), abdominal bloating (OR 175.3, 95\% Cl 28.4-1719.9) and abdominal pain (OR 84.1, 95\% Cl 0.8-8845.6) figure 1-5. Borborygmi (OR 3.6, 95\% $\mathrm{Cl} 0.3-$ 33-4) and diarrhea showed no significance in postprandial symptom generation (OR 6.2, 95\% Cl 0.0-1126.2). All results were adjusted for age and gender.

In the FD-IBS subgroup there was a significant difference in LHBT induced nausea (OR 37.6, 95\% Cl 1.9-34019.4), abdominal bloating (OR $527.095 \% \mathrm{Cl}$ 117.6-3281.4), abdominal pain (OR 286.1, 95\% Cl 31.9-2564.6) and borborygmi (OR 13.3, 95\% $\mathrm{Cl} 3.2-61.6$ ) compared to HV figure 1-5. A significant effect on diarrhea was not found (OR 14.8, 95\% Cl 0.2-1124.7). Again, results remained unchanged after adjustment for age and gender.

In the subgroup of IBS-only compared to HV there was a significant difference in LHBT induced abdominal pain (OR 161.2, 95\% Cl 16.9-1534.8), abdominal bloating (OR 524.1, 95\% Cl 114.73432.3), borborygmi (OR 17.7, 95\% $\mathrm{Cl} 4.7-67.4$ ) and diarrhea (OR 25.8, 95\% Cl 2.0-7012.6). No significant difference was seen in nausea (OR 15.5, 95\% Cl 1.0-16267.8).

\section{Time effect}

There was a significant effect of time in FD-only patients on nausea (OR $0.87,95 \% \mathrm{Cl} 0.82$ 0.92), borborygmi (OR 1.14, 95\% Cl 1.07-1.23), diarrhea (OR 1.28, 95\% Cl 1.10-1.55), but not on abdominal bloating (OR 1.04, 95\% $\mathrm{Cl} 0.99-1.09$ ) and pain (OR 0.98, 95\% $\mathrm{Cl} 0.93-1.04$ ). 
The time effect in FD-IBS patients was significant for nausea (OR 0.87, 95\% $\mathrm{Cl} 0.83-0.90$ ), abdominal bloating (OR 1.09 95\% Cl 1.06-1.13), borborygmi (OR 1.11, 95\% Cl 1.07-1.15), diarrhea (OR1.16, 95\% Cl 1.09-1.24), but not for abdominal pain (OR 1.03, 95\% Cl 1.00-1.07). In IBS-only patients there was a significant effect of time on abdominal bloating (OR 1.13, 95\% $\mathrm{Cl}$ 1.11-1.16), on diarrhea (OR 1.26, 95\% Cl 1.21-1.32), borborymgi (OR 1.10, 95\% Cl 1.07-1.13), in nausea (OR 0.86, 95\% $\mathrm{Cl} 0.83-0.89$ ) and in abdominal pain (OR 1.08, 95\% $\mathrm{Cl} 1.05-1.10$ ).

\section{Hydrogen Values}

\section{Group effect}

FD patients averaged 2.3ppm higher hydrogen values $(95 \% \mathrm{Cl}(-2.7)-7.2)$ than $\mathrm{HV}$, without reaching significance. The effect of FD-only and FD-IBS subgroups on hydrogen values was neither significant considering the whole period (10-180 minutes) with FD-only patients having $0.2 \mathrm{ppm}$ and FD-IBS patients having $2.9 \mathrm{ppm}$ higher hydrogen values compared to HV (95\% Cl (-6.1)-6.5 resp. (-2.4)-8.2). IBS patients had an average of 3.59ppm higher hydrogen values $(95 \% \mathrm{Cl}(-1.36)-8.55)$ than $\mathrm{HV}$. figure 6

After adjusting for age and gender the difference in hydrogen values between FD/IBS and HV remained insignificant $(2.4,95 \% \mathrm{Cl}(-2.9)-7.6$ / 2.65, 95\% $\mathrm{Cl}(-1.49)-7.79)$.

\section{Discrimination between FD patients and HV}

There was a good discriminatory capacity for FD and IBS-only as compared to HV (AUC $=0.82$ resp. $A U C=0.91$ ) and a high predictive capacity for FD (sensitivity $=0.72$, specificity $=0.84$ ) based on abdominal symptoms and hydrogen values. In FD-only patients AUC was 0.83 (sensitivity $=0.69$, specificity $=0.88$ ), in FD-IBS 0.84 (sensitivity $=0.84$, specificity $=0.80$ ) and in IBS-only sensitivity was 0.91 and specificity $=0.56$. figure $7-10$

\section{Discussion}

This study demonstrates a significant difference in LHBT induced abdominal symptoms in patients with FD and IBS compared to HV. In specific, abdominal pain, bloating, borborygmi and nausea were significantly more often induced in FD patients than HV, with a test that was originally developed to discriminate IBS patients from HV (8). It is currently believed that visceral hypersensitivity of the upper GI tract is partly accountable for these findings in FD 
patients. (9). In a recent review, the impaired somatosensation in FD patients was attributed to neuro-imaging anomalies in the somatosensory cortex, the cognitive and the affective regions. These, in turn, were assumed to be involved in a dysfunctional gut-brain axis thereby leading to altered symptom perception in FD patients (10). In accordance to this, Farre et al. demonstrated significantly lower postprandial discomfort thresholds during balloon distention in FD patients compared to controls and attributed this to visceral hypersensitivity (11). However, balloon distention tests are neither practical nor well tolerated in patients nor widely available and constitute an assessment reserved for research or highly specialized centers. This underlines the importance of analyzing responses of standardized foodprovocation testing in the large patient group with disorders of gut-brain interaction. A previous study by Woodtli et al. (12) addressing FD in combination with food intake showed induction of nausea and bloating after lipid but not saline infusion in FD patients. These findings go in line with our results showing induction of bloating and nausea after ingestion of our liquid lipid-containing test meal in FD patients. It leads to the assumption of duodenal hypersensitivity to lipids and consecutive symptom generation in FD patients but not HV. It also supports the hypothesis of the test meal originally developed for IBS diagnostics representing an adequate composition or at least containing important ingredients for FD diagnostics, as well. Consequently, the advantage of the studied test method, as compared to previous FD studies is, that the same substances and setting can as well be used for IBS patients. In contrast to other nutrient studies in FD patients our test uses a significantly higher caloric load (13). This might explain the clear discrimination between FD, IBS and HV based on abdominal symptoms. Another advantage is, that no detailed questionnaire needs to be completed to discriminate between FD/IBS patients and HV as it has been the case for previous patient-reported outcome measures (PROM) (14-17). The test also meets the FDA criterion of a PROM representing a direct report from the patient without its interpretation by a clinician. In the past, tests with investigator-administered instruments were described to be limited as clinician's interpretation often underestimates symptom severity $(18,19)$.

A limitation of our study is that our symptom assessment included only nausea, abdominal bloating and abdominal pain as typical FD symptoms. We did not document symptoms of early satiation, postprandial fullness or belching. However, the few symptoms were sufficient to discriminate between FD and HV with high specificity. 
In contrast to the significant difference in abdominal symptoms, no significant hydrogen difference was seen in FD/IBS as compared to HV. However, hydrogen values tended to be higher in FD/IBS patients compared to HV between 30-100min figure 6. A possible explanation for this might be a higher mucosal bacterial load in the proximal small intestine. This hypothesis would be consistent with the finding of Zhong et al. who showed a positive correlation between duodenal mucosal bacterial load and symptom severity response to a standardized meal in FD patients $(20,21)$, including increase in total duodenal bacterial load. Supporting the involvement of the microbiome in FD patients is the fact that a large proportion of FD patients in the underlying cohort suffered from FD-IBS overlap. A higher prevalence of small intestine bacterial overgrowth (SIBO) is known in IBS patients and therefore likely in IBS-FD patients, too (22). However, the IBS influence only partially explains this finding since also patients with isolated FD tended to have higher hydrogen values figure 6. Costa et al. postulated that also FD patients could have a higher prevalence of SIBO compared to HV (23) based on higher hydrogen measurements after lactulose ingestion.

If the LHBT does not only allow to discriminate between FD/IBS and HV but also predicts response to different FD or IBS therapies has not been evaluated yet. As lactulose technically belongs to the FODMAP group (fermentable oligo-, di-, monosaccharides and polyols), one could assume that FD and IBS patients with increased abdominal symptoms induced by LHBT might respond better to a diet low in FODMAPs. However, lactulose may only represent one FODMAP-subgroup. It has yet to be shown if other FODMAP-groups induce similar symptom patterns and potentially predict treatment response. To verify this, prospective studies are needed.

In conclusion, the underlying analysis demonstrated significant differences in abdominal symptoms induced by LHBT in FD patients compared to HV, suggesting a practical and safe method to not only distinguish between IBS and HV as described by Le Neve et al. but also between FD and HV. Additionally, despite big efforts during the last years no developed PROM is currently considered appropriate in FD patients (17). Considering this, we think, that the LHBT as an instrument including subjective as well as objective measure represents a useful tool for diagnostic work-up and to monitor and predict the therapeutic progress in FD patients in the future. 



\section{References}

1. Stanghellini V, Talley NJ, Chan F, Hasler WL, Malagelada J, Suzuki H, et al. Rome IV Gastroduodenal Disorders. Gastroenterology. 2016.

2. Bisschops R, Karamanolis G, Arts J, Caenepeel P, Verbeke K, Janssens J, et al. Relationship between symptoms and ingestion of a meal in functional dyspepsia. Gut. 2008;57(11):1495-503.

3. Taylor F, Reasner DS, Carson RT, Deal LS, Foley C, lovin R, et al. Development of a SymptomBased Patient-Reported Outcome Instrument for Functional Dyspepsia: A Preliminary Conceptual Model and an Evaluation of the Adequacy of Existing Instruments. Patient. 2016;9(5):409-18.

4. Rezaie A, Buresi M, Lembo A, Lin H, McCallum R, Rao S, et al. Hydrogen and Methane-Based Breath Testing in Gastrointestinal Disorders: The North American Consensus. Am J Gastroenterol. 2017;112(5):775-84.

5. Le Neve B, Brazeilles R, Derrien M, Tap J, Guyonnet D, Ohman L, et al. Lactulose Challenge Determines Visceral Sensitivity and Severity of Symptoms in Patients With Irritable Bowel Syndrome. Clin Gastroenterol Hepatol. 2016;14(2):226-33 e1-3.

6. Pohl D, Van Oudenhove L, Törnblom H, Le Nevé B, Tack J, Simrén M. Functional Dyspepsia and Severity of Psychologic Symptoms Associate With Postprandial Symptoms in Patients With Irritable Bowel Syndrome. Clinical Gastroenterology and Hepatology.

7. Tack J, Talley NJ, Camilleri M, Holtmann G, Hu P, Malagelada JR, et al. Functional gastroduodenal disorders. Gastroenterology. 2006;130(5):1466-79.

8. Le Neve B, Posserud I, Bohn L, Guyonnet D, Rondeau P, Tillisch K, et al. A combined nutrient and lactulose challenge test allows symptom-based clustering of patients with irritable bowel syndrome. Am J Gastroenterol. 2013;108(5):786-95.

9. Vanheel H, Carbone F, Valvekens L, Simren M, Tornblom H, Vanuytsel T, et al. Pathophysiological Abnormalities in Functional Dyspepsia Subgroups According to the Rome III Criteria. The American Journal Of Gastroenterology. 2016;112:132.

10. Lee IS, Wang $H$, Chae $Y$, PreissI H, Enck P. Functional neuroimaging studies in functional dyspepsia patients: a systematic review. Neurogastroenterol Motil. 2016;28(6):793-805.

11. Farre R, Vanheel $\mathrm{H}$, Vanuytsel T, Masaoka T, Tornblom H, Simren M, et al. In functional dyspepsia, hypersensitivity to postprandial distention correlates with meal-related symptom severity. Gastroenterology. 2013;145(3):566-73.

12. Woodtli W, Owyang C. Duodenal pH governs interdigestive motility in humans. Am J Physiol. 1995;268(1 Pt 1):G146-52.

13. Parker HL, Tucker E, Blackshaw E, Hoad CL, Marciani L, Perkins A, et al. Clinical assessment of gastric emptying and sensory function utilizing gamma scintigraphy: Establishment of reference intervals for the liquid and solid components of the Nottingham test meal in healthy subjects. Neurogastroenterol Motil. 2017;29(11).

14. Rentz AM, Kahrilas P, Stanghellini V, Tack J, Talley NJ, de la Loge C, et al. Development and psychometric evaluation of the patient assessment of upper gastrointestinal symptom severity index (PAGI-SYM) in patients with upper gastrointestinal disorders. Qual Life Res. 2004;13(10):1737-49.

15. Talley NJ, Haque M, Wyeth JW, Stace NH, Tytgat GN, Stanghellini V, et al. Development of a new dyspepsia impact scale: the Nepean Dyspepsia Index. Aliment Pharmacol Ther. 1999;13(2):22535 .

16. Shaw M, Talley NJ, Adlis S, Beebe T, Tomshine P, Healey M. Development of a digestive health status instrument: tests of scaling assumptions, structure and reliability in a primary care population. Aliment Pharmacol Ther. 1998;12(11):1067-78.

17. Smeets FGM, Masclee AAM, Conchillo JM, Keszthelyi D. Systematic review: Disease-specific instruments to assess gastrointestinal symptoms in functional dyspepsia. Neurogastroenterol Motil. 2018.

18. Fransen GA, Janssen MJ, Muris JW, Mesters I, Knottnerus JA. Measuring the severity of upper gastrointestinal complaints: does GP assessment correspond with patients' self-assessment? Fam Pract. 2007;24(3):252-8. 
19. Madsen LG, Hansen JM, Gronvold M, Bytzer P. The validity of a symptom diary in ratings of dyspepsia measured against a detailed interview: do patients and clinicians agree in their assessment of symptoms? Aliment Pharmacol Ther. 2007;26(6):905-12.

20. Zhong L, Shanahan ER, Raj A, Koloski NA, Fletcher L, Morrison M, et al. Dyspepsia and the microbiome: time to focus on the small intestine. Gut. 2017;66(6):1168-9.

21. Walker MM, Talley NJ. The Role of Duodenal Inflammation in Functional Dyspepsia. Journal of Clinical Gastroenterology. 2017;51(1):12-8.

22. Posserud I, Stotzer P-O, Björnsson ES, Abrahamsson H, Simrén M. Small intestinal bacterial overgrowth in patients with irritable bowel syndrome. Gut. 2007;56(6):802-8.

23. Costa MB, Azeredo Jr IL, Marciano RD, Caldeira LM, Bafutto M. Evaluation of small intestine bacterial overgrowth in patients with functional dyspepsia through $\mathrm{H} 2$ breath test. Arq Gastroenterol. 2012;49(4):279-83.

24. Walker MM, Talley NJ. Review article: bacteria and pathogenesis of disease in the upper gastrointestinal tract--beyond the era of Helicobacter pylori. Aliment Pharmacol Ther. 2014;39(8):767-79.

25. Tan VPY, Liu KSH, Lam FYF, Hung IFN, Yuen MF, Leung WK. Randomised clinical trial: rifaximin versus placebo for the treatment of functional dyspepsia. Alimentary Pharmacology \& Therapeutics. 2017;45(6):767-76. 
Figure 1: mean level of abdominal pain in patients with $\mathrm{FD} / \mathrm{IBS}$ and $\mathrm{HV}$ over time. $\mathrm{HC}$ : healthy controls, FD: patients with functional dyspepsia, IBS only: patients with only irritable bowel syndrome, FD only: patients with only functional dyspepsia, FD-IBS overlap: patients with functional dyspepsia and irritable bowel syndrome overlap

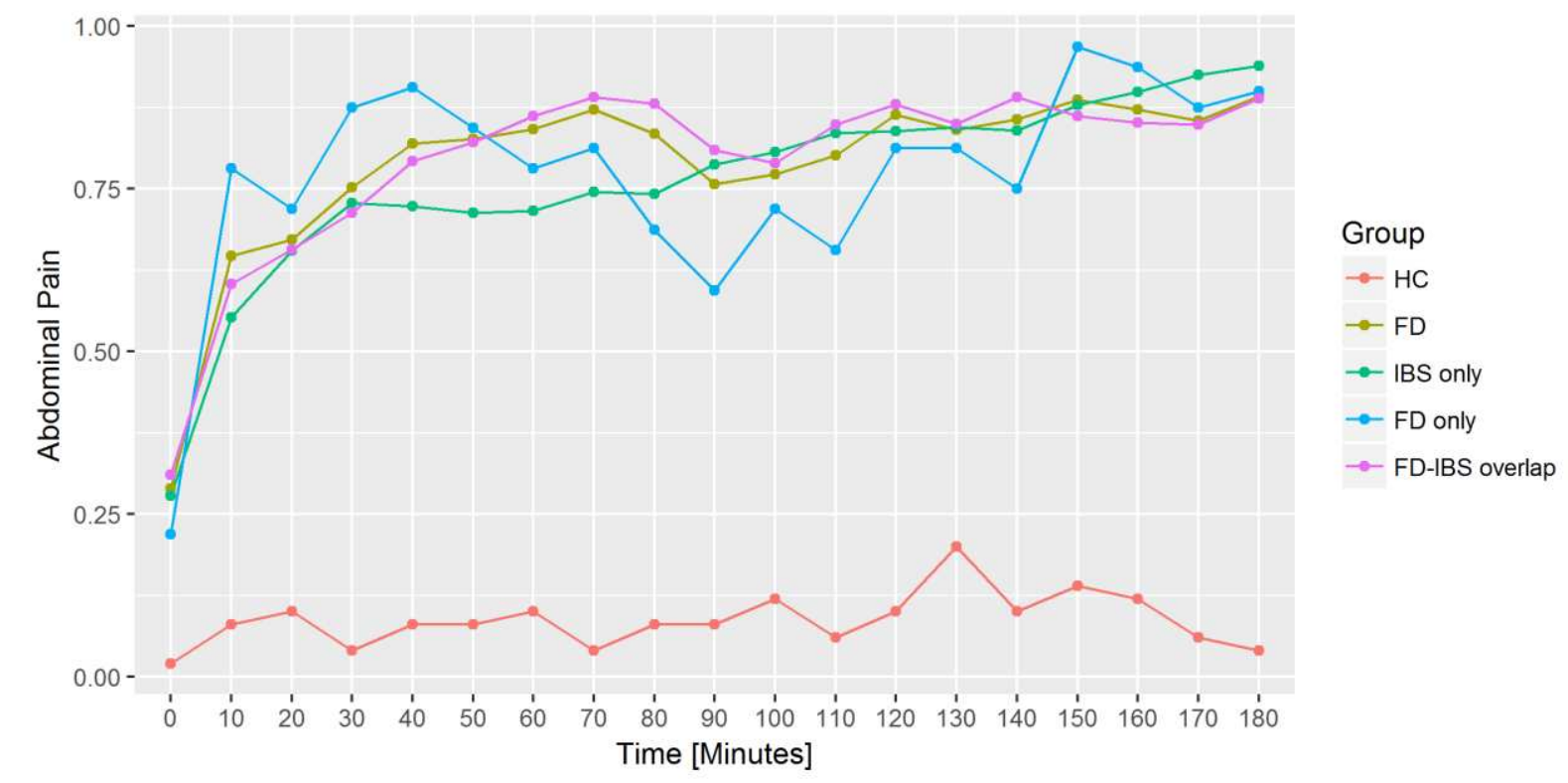


Figure 2: mean level of abdominal bloating in patients with FD/IBS and HV over time. HC: healthy controls, FD: patients with functional dyspepsia, IBS only: patients with only irritable bowel syndrome, FD only: patients with only functional dyspepsia, FD-IBS overlap: patients with functional dyspepsia and irritable bowel syndrome overlap

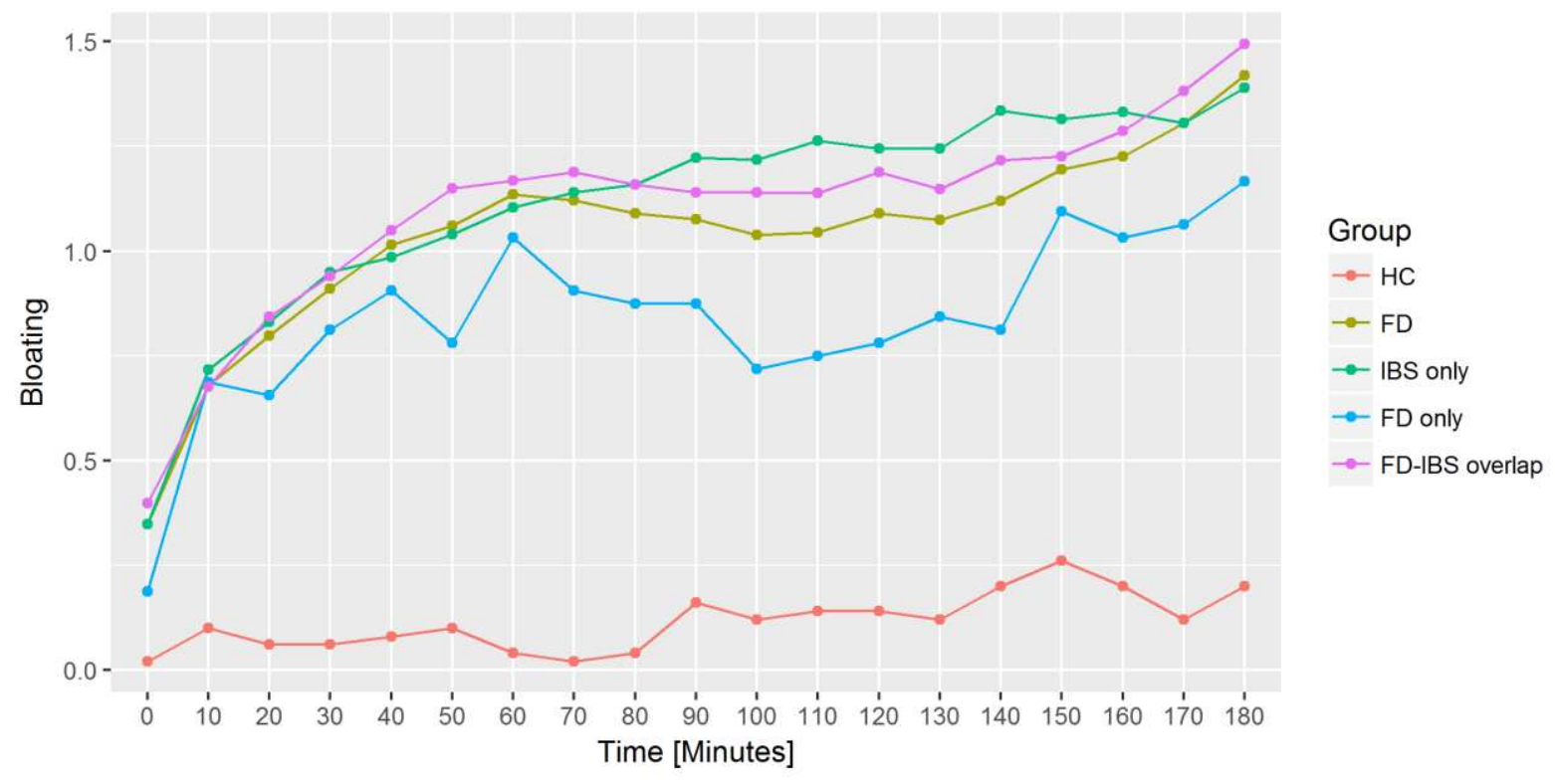


Figure 3: mean level of borborygmi in patients with FD/IBS and HV over time. HC: healthy controls, FD: patients with functional dyspepsia, IBS only: patients with only irritable bowel syndrome, FD only: patients with only functional dyspepsia, FD-IBS overlap: patients with functional dyspepsia and irritable bowel syndrome overlap

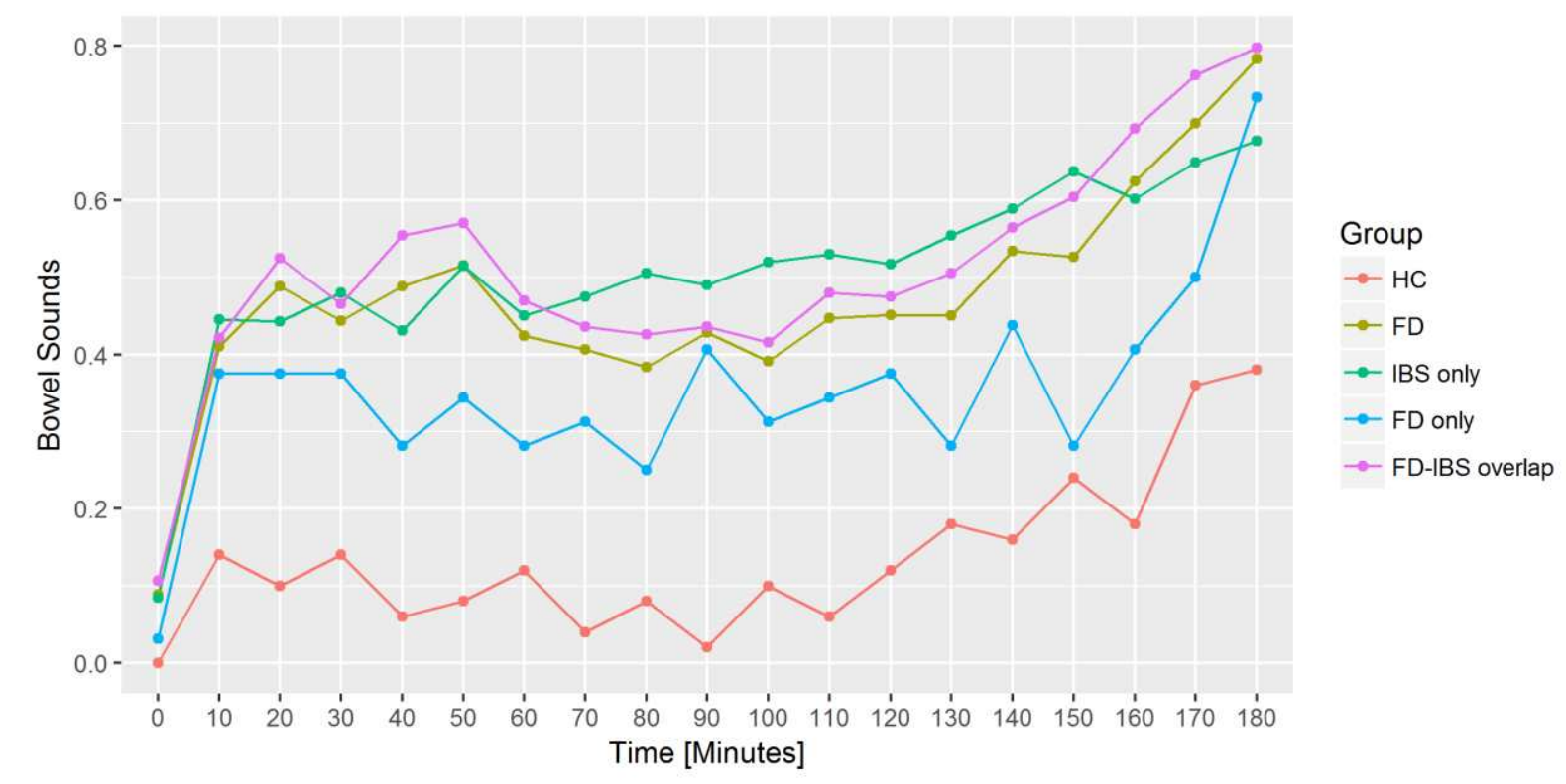


Figure 4: mean level of nausea in patients with FD/IBS and HV over time. HC: healthy controls, FD: patients with functional dyspepsia, IBS only: patients with only irritable bowel syndrome, FD only: patients with only functional dyspepsia, FD-IBS overlap: patients with functional dyspepsia and irritable bowel syndrome overlap

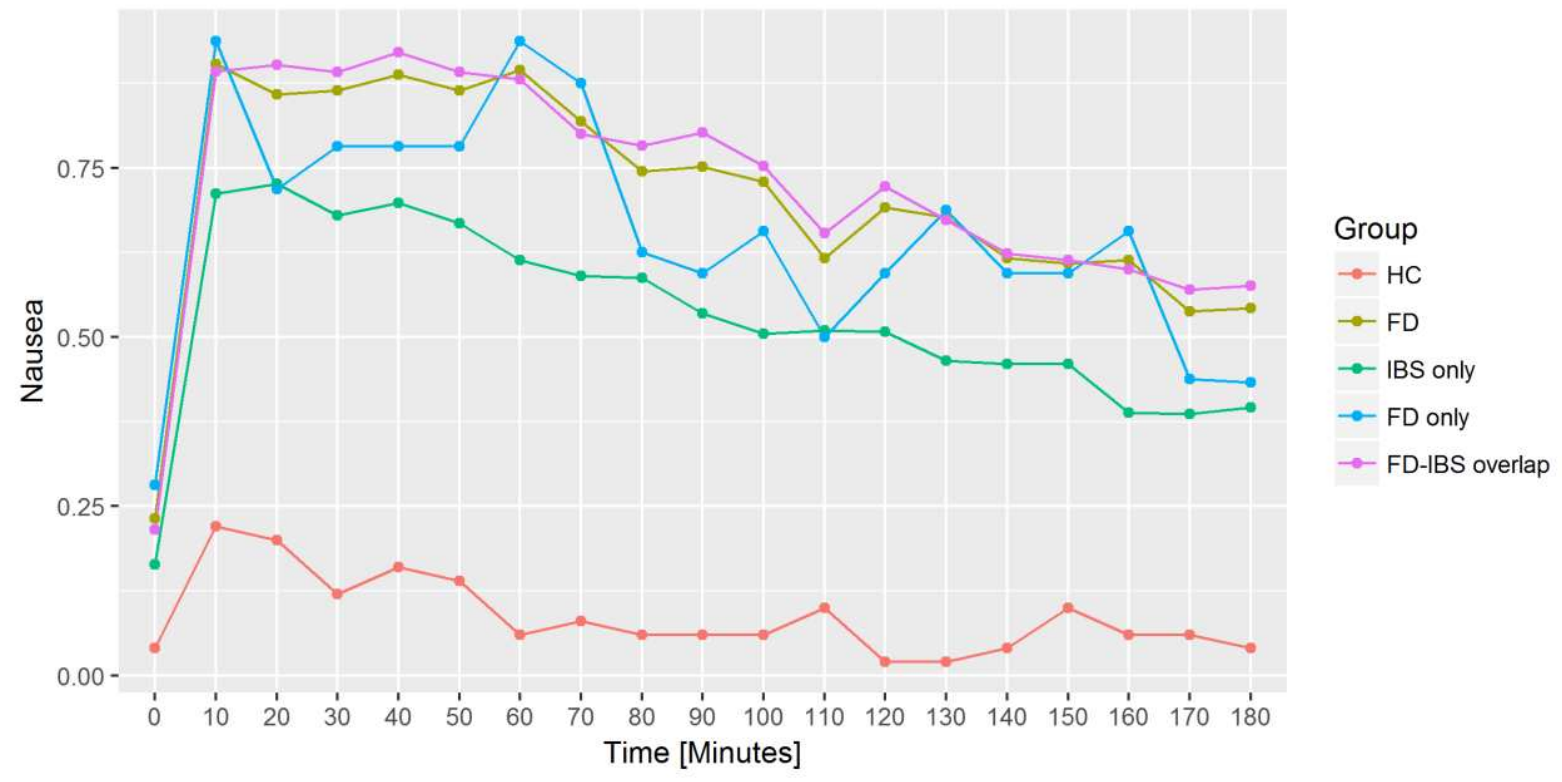


Figure 5: mean level of diarrhea in patients with FD/IBS and HV over time. HC: healthy controls, FD: patients with functional dyspepsia, IBS only: patients with only irritable bowel syndrome, FD only: patients with only functional dyspepsia, FD-IBS overlap: patients with functional dyspepsia and irritable bowel syndrome overlap

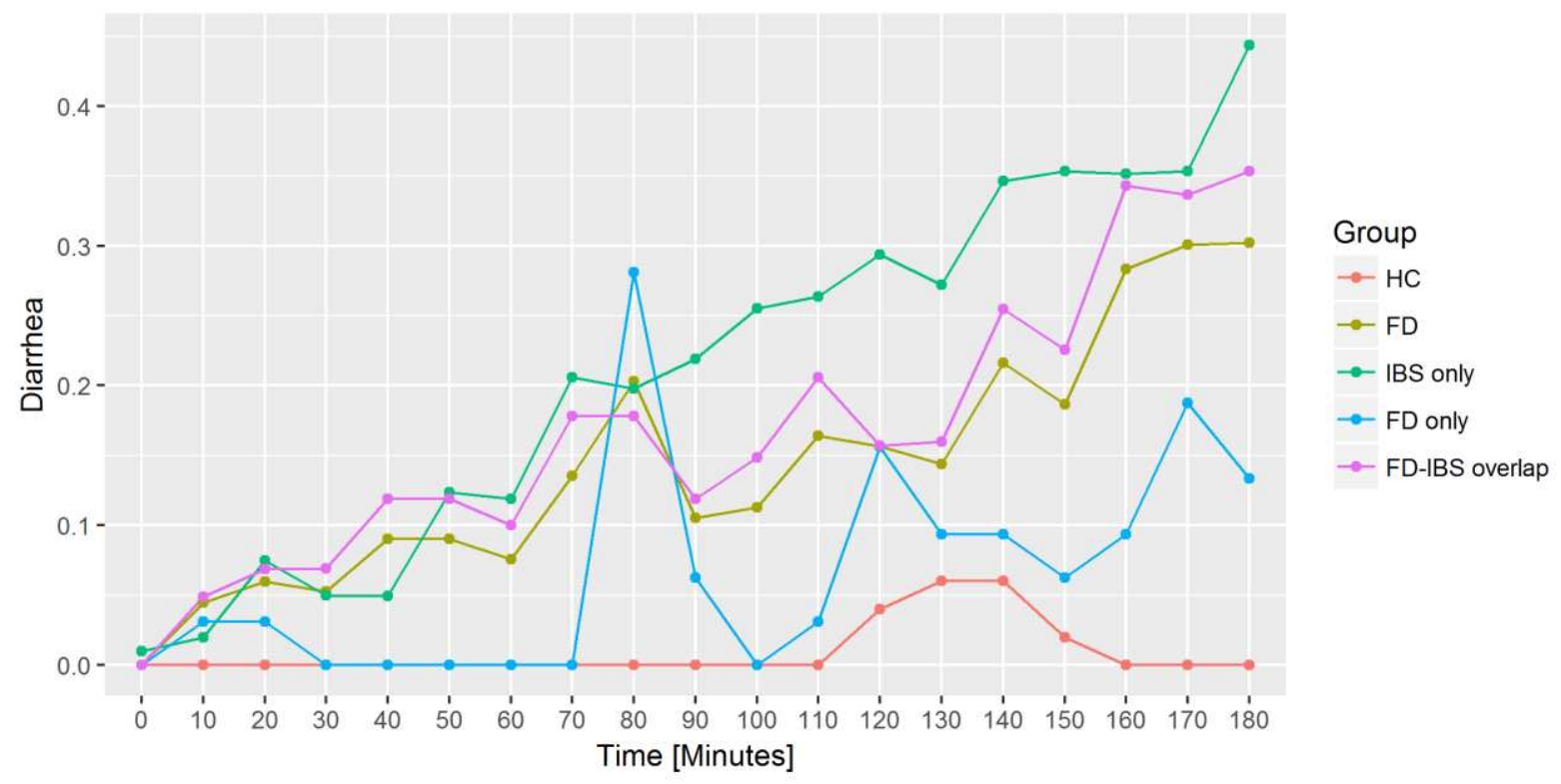


Figure 6: mean level of hydrogen in patients with FD/IBS and HV over time. HC: healthy controls, FD: patients with functional dyspepsia, IBS only: patients with only irritable bowel syndrome, FD only: patients with only functional dyspepsia, FD-IBS overlap: patients with functional dyspepsia and irritable bowel syndrome overlap

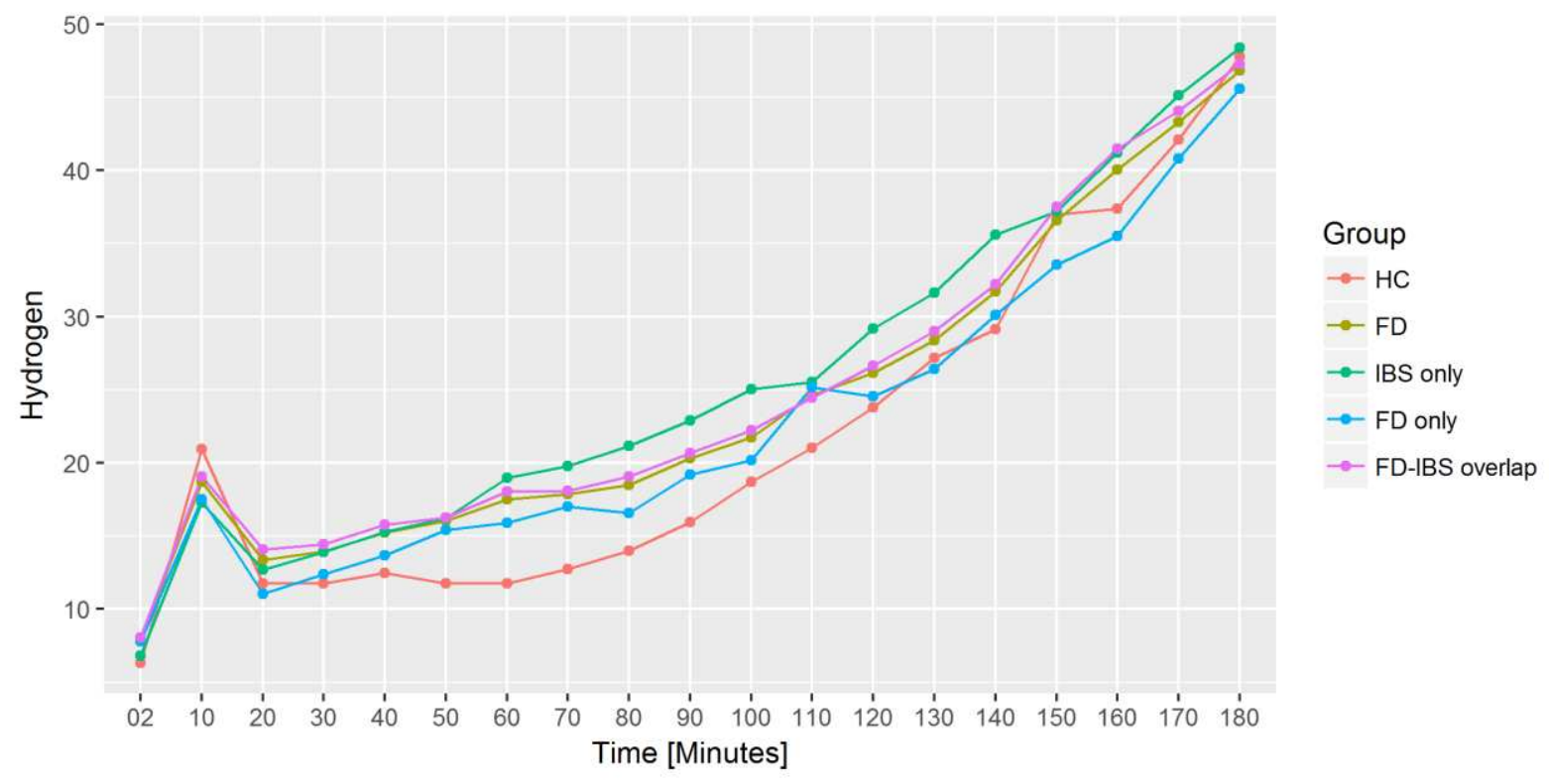


Figure 7: Receiver Operating Characteristic Curve (ROC) for the median of abdominal symptoms and hydrogen values in functional dyspepsia (FD) patients compared to healthy controls (HC)

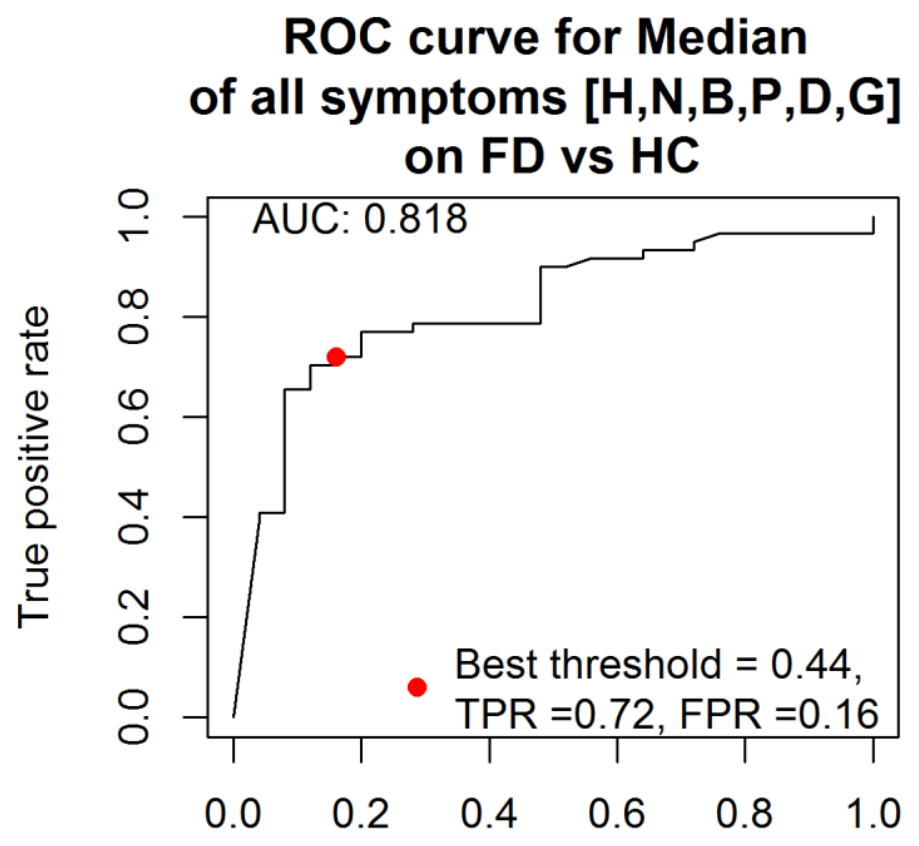

False positive rate 
Figure 8: Receiver Operating Characteristic Curve (ROC) for the median of abdominal symptoms and hydrogen values in functional dyspepsia (FD-only) patients compared to healthy controls (HC)

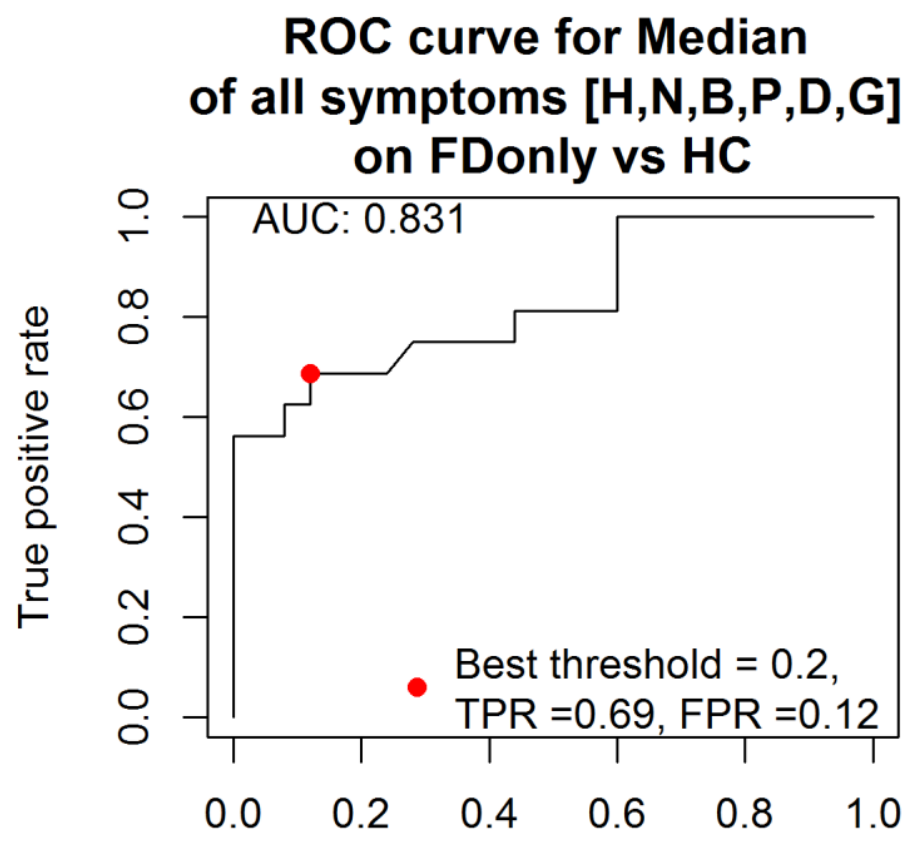

False positive rate 
Figure 9: Receiver Operating Characteristic Curve (ROC) for the median of abdominal symptoms and hydrogen values in mixed type functional dyspepsia and irritable bowel syndrome (FD-IBS) patients compared to healthy controls (HC)

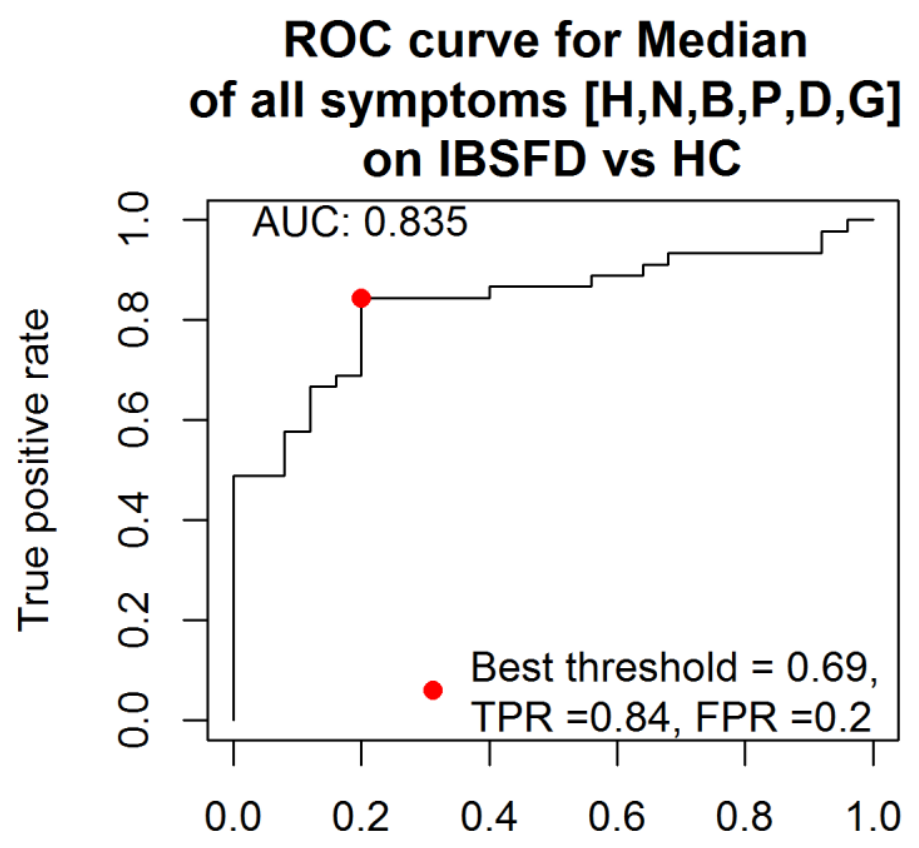

False positive rate 
Figure 10: Receiver Operating Characteristic Curve (ROC) for the median of abdominal symptoms and hydrogen values in irritable bowel syndrome (IBS-only) patients compared to healthy controls (HC)

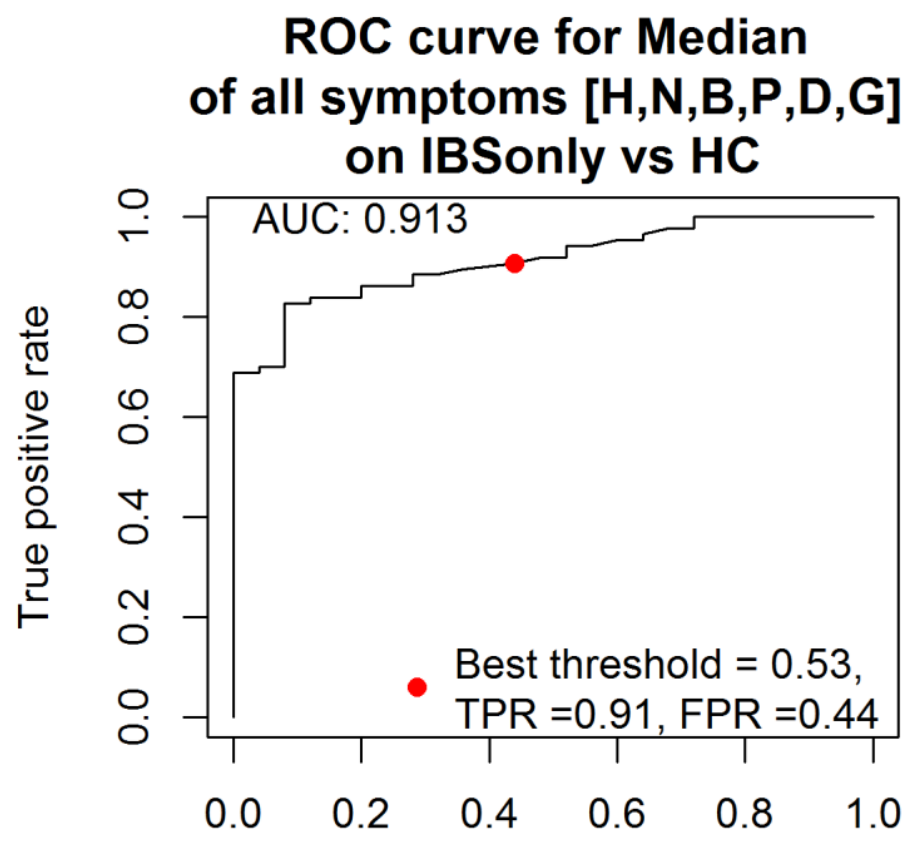

False positive rate 